and lizards-dactylosoma and cytamœba-do not. He uses this as an argument against regarding exflagellation as a necessary and vital process in the malaria parasite of man. I regard it, on the contrary, as an argument in favour of my hypothesis. Mosquitos do not prey on frogs and lizards; therefore the gymnosporidia of these cold-blooded reptiles must find some other way of continuing their species than that applying to the gymnosporidia of man and birds ; and if it be so, why not by a different adaptation of the parasite concerned?

It has also been advanced that although the malaria parasites of birds flagellate, this flagellation cannot be a vital process adapted to such conditions as the mosquito supplies, seeing, it is said, that mosquitos do not attack birds. As a matter of fact, I know that mosquitos do prey on birds in China, and Lewis has had a similar experience in India.

The most Serious Orjection to the Mosquito Theory.

To my way of thinking the most serious objection to the mosquito theory lies in the fact that as yet we have not been able ito trace the flagellum into the tissues of the mosquito. The reasons for this I believe to be the delicacy and minuteness of the object, the complicated character and abundance of cell forms, with which we are as yet very imperfectly acquainted, in the mosquito, and our ignorance of a suitable technique. Until the flagellum has been traced into some cell in the mosquito it is always possible that I have been misled by the marvellous analogy between the requirements and structure of the plasmodium and those of the filaria, and by the apparent suitability of the structure and habits of the flagellated plasmodium and the flagellum to the conditions they encounter in the mosquito. It is just possible that the invariableness with which the metamorphosis of the crescent is effected in the mosquito, the certainty with which "the manifestation " of flagellation occurs in from twenty to thirty minutes after ingestion of malarial blood by the mosquito, are simply coincidence. One may say, the phagocytes live for a time in the mosquito, why not the flagellated body? It can live on the microscope slide, why should it not live in the mosquito's stomach?

Confirmation of the Theory Anticipated.

While acknowledging that these are points requiring further investigation, I do not recognise in them grave arguments against the validity of my theory. I do not hold that the theory is established, but I do hold that Ross's investigations have rendered it so probable that it is in a fair way of being established; and I look confidently to him and to other workers in India and elsewhere to forge the links that are still wanting to complete the chain of evidence.

Scientific and Practical Developments Likely to AcCRUE.

If it is established, as I believe that ere long it will be, that the plasmodium is a parasite of the mosquito, that malaria is a disease of insects, we may look for some strange scientific and practical developments. The discovery will spread before us a vast field for future study. The real science of malaria will only be begun. We may find that the special forms of the plasmodium-the quartan, the tertian, and the malignant-each requires a special species of mosquito host. We know that special localities have special clinical types of malaria. May not this depend on local peculiarities as regards species of mosquito? The different species of mosquito will require to be determined, their special habits ascertained, and the action of each as regards the plasmodium studied. We may thus be enabled to ascertain one reason why some tropical regions are malarious whilst others are not. We may even find a way of controlling malaria by introducing the natural enemies of the malaria germ into malarious localities-cats to keep down rats. We will be able to settle the long-vexed question as to the possibility of contracting malaria from drinking water, the exact physical conditions that favour and regulate the diffusion of the malaria germ in the atmosphere, and many other points of important practical bearing

Precision, too, will in time be given by such studies to our efforts at prophylaxis in malaria and malarial disease, efforts which at present are but vaguely directed. The hygiene of malarious localities will become a matter of science instead of, as at present, mere haphazard empiricism founded on what is little better than tradition.

\section{A Sugaestion.}

I understand that extensive drainage works are about to be undertaken in India with the object of mitigating the insalubrity of certain pestiferous districts, and that the schemes entail an enormous expenditure of the public money. This money will be expended simply to fight the plasmodium. Would it not be well to know a little more about the enemy before attacking him? Would it not be a wise, a businesslike thing, before proceeding to expend powder and shot, first to find out something about his habits, and so learn when, and where, and how he may be most easily, most effectually, and most economically attacked? I think the Indian Government would do well to do this, and to encourage men like Ross in their efforts to lay a scientific foundation on which a truly scientific prophylaxis might subsequently be built.

\section{TEN CASES OF OPERATION FOR EXTRA- UTERINE FETATION.}

By HARRISON CRIPPS, F.R.C.S.,

Assistant Surgeon, St. Bartholomew's Hospital, and Operator for Abdominal Sections in the Women's Ward.

IT has not yet been established that extrauterine fotation. commences anywhere except in some portion of the Fallopian. tube. By bearing in mind the relation of this structure to the broad ligament it will be understood how different the conditions will be according as to whether the fotal sac as it grows protrudes upwards or downwards. If upwards, it will protrude like a sacculated aneurysm into the abdominal cavity; if downwards, it will extend between the layers of the broad ligament, separating them widely. When rupture takes place through the free surface of the sac there is nothing to arrest the hæmorrhage, and a large number of cases bleed to death in a few hours. On the other hand, when the blood is poured downwards between the layers of the broad ligament it will spontaneously cease, as these can only be detached to a limited extent. In a large number of these latter cases the extravasated blood ultimately becomes absorbed and the patient recovers. There is, however, a third condition, and which is probably more common than eitherof the other two (see Cases III and v), in which a ruptured tube pours out blood in such a way that it becomes encysted in Douglas's pouch. In these cases a mass of coagulated: blood forms a tumour the size of a cocoanut, bounded below and at the sides by Douglas's pouch, and above by the dilated tube, small intestine, and free end of the omentum bound together by inflammation and plastered over the roof of the pelvis. The probable explanation of these intraperitoneal circumscribed collections is that some localised peritonitis is set up in the first place by a slight oozing or irritation, and thus the general cavity of the peritoneum is shut off before any extensive pouring out of blood occurs.

Without recapitulating all the well-known symptoms which are so diagnostic of ruptured extrauterine pregnancies, it may be worth while to mention some of the chief points in the differential diagnosis between rupture occurring free into the peritoneal cavity, or when it occurs between the layers of the broad ligament, or when circumscribed in Douglas's pouch, for the whole question of operative treatment depends upon the position of the rupture.

When the sac bursts into the peritoneum, pain, though severe, is not so intense as when the bleeding is into the broad ligament. On the other hand, the general symptoms are graver, doubtless due to a larger amount of blood being lost. Indeed, the hæmorrhage may be so profuse, that within an hour or two of the accident the patient may be pulseless, and almost moribund, but as a rule she will rally to a certain extent from the immediate effects of the first loss of blood, thus affording time for surgical interference, which, to be effective, must be prompt, the cases being almost invariably fatal if the bleeding is not arrested by abdominal section. 
The general symptoms of shock such as is produced by loss of blood from any part are most marked. The abdomen is tender ; it is resonant in front, and often there is considerable impairment of resonance, or even dulness in the flanks. Careful palpation of the abdomen fails to detect any obvious tumour. On making a vaginal examination the uterus is movable, and in the natural position. No tumour can be felt, but there may be some fulness in Douglas's pouch conveying a doughy feeling to the finger, doubtless due to bloodclot. Examination of the rectum shows no stricture of the bowel.

If the rupture has occurred between the layers of the broad ligament the sudden onset of the symptoms will be the same, but the pain seems to be more intense and lasting, with a well-marked paroxysmal character described by patients as like labour pains. The patient generally shows signs of hæmorrhage, though in a much less degree than in the intraperitoneal cases. On deep abdominal palpation a tumour feeling hard may be found in one or other side rising out of the pelvis, though not to a great extent. Sometimes the swelling does not rise sufficiently to be obvious on abdominal examination. It is on examining by the vagina, however, that the more important points in the differential diagnosis are to be obtained, for in intraperitoneal rupture there is nothing abnormal to be felt, save perhaps the puffy swelling already alluded to in Douglas's pouch, whereas, in the broad ligament variety a hard swelling can be felt on one or other side of the cervix partly filling the pelvic cavity. The tumour is firmly fixed, while the uterus pushed to one side appears to form the inner boundary of the swelling, and is attached to it. On examining the rectum a marked constriction is often present at about 4 inches from the anus; this is due to the rectal fascia, which passes round the bowel and is continuous with some of the broad ligament fibres. "Hence, if the broad ligament is pulled upon as it is when distended by an effusion between its layers, the rectal fascia becomes drawn tighter, ,producing a ring-like stricture.

Diagnosis when the Rupture is into Douglas's Pouch. I believe this to be, perhaps, the commonest form of rupture, and I have certainly met several instances of it. In these cases, owing to a slight oozing, which at first takes place, and which is the probable cause of the localised peritonitis which ultimately limits the effusion, there may be an absence of the sudden acute pain followed by collapse which is so characteristic of the other two varieties of rupture already mentioned. The general history suggestive of a pregnancy may be present, together with some pain, though not of an acute nature, about the pelvis. An examination per vaginam discloses a swelling, perhaps the size of a cocoanut, and this lies behind the uterus in Douglas's pouch. The swelling is elastic, feeling like an ovarian cyst, for which it is, indeed, generally mistaken.

In cases in which $I$ had an opportunity of making a thorough examination the swelling did not feel nearly as hard as if the blood had been in the broad ligament. The swelling, too, chiefly occupies the middle line rather than one side or the other. The uterus, although its mobility is impaired, is not so firmly fixed as in the broad ligament cases, and there is no stricture of the rectum.

\section{Question of Operation in Ruptured Extrauterine} F'aETATION.

This must be considered, first, in those cases where the rupture has been intraperitoneal, and, secondly, where it has ruptured into the broad ligament or in which it is limited to Douglas's pouch.

Rupture into the Peritoneal Cavity.

If left alone these cases almost invariably die from hæmorrhage, and there can be no question, in the light of modern experience, that directly the condition is recognised the abdominal cavity should be opened, and the bleeding points secured by ligature. In the whole range of abdominal surgery no more brilliant results are obtained than thus treating a ruptured tube. Without operation, not only is death nearly certain, but it occurs with tragic rapidity. On the other hand, by a prompt operation the large majority of the cases are saved, and in the course of a few weeks restored to perfect health.

Rupture into the Broad Ligament.

When the hæmorrhage is limited to the broad ligament or to Douglas's pouch, a grave question arises as to the propriety of surgical interference.

There can be no doubt that in a large number of cases where the bleeding is into the broad ligament, the patients get perfectly well without interference. The death of the fœetus seems coincident with the hæmorrhage. The bleeding, being limited by the broad ligament, is not sufficient to be fatal, and in the course of a few weeks will disappear by absorption with the recovery of the patient. No doubt, in some of these cases a secondary rupture of the distended broad ligament may occur, and a fatal hæmorrhage thus takes place into the abdomen. Again, it is possible that the fœtus may not be killed, and may go on developing in the broad ligament, while in other cases suppuration, with its consequent troubles, will result from the extravasated clot.

These occurrences, however, seem to be so exceptional that their anticipation does not justify an operation; so that, if there is no evidence of any increase of the swelling, and if the general symptoms do not indicate a continuance of the bleeding, such cases are best left alone, merely keeping the patient absolutely at rest in the recumbent position. On the other hand, if the symptoms of hæmorrhage continue, or if the swelling which may have remained stationary for some days again increases in size with an exacerbation of pain, an operation should be undertaken.

Cases will occur in which, owing to the absence of any indication pointing strongly one way or another, a legitimate doubt must arise as to the propriety of interfering. In these circumstances the surroundings of the patient must be taken into consideration when forming a decision. In a hospital or well-appointed house with sanitary surroundings, a good nurse, and a surgeon accustomed to abdominal sections I would advise an operation, while without these advantages in a doubtful case it would be wiser to leave things alone.

H zemorrhage limited to Douglas's Pouch.

There has yet to be considered the propriety of operating where the hæmorrhage is presumed to be into Douglas's pouch and limited by peritoneal adhesions above, as already described. The diagnosis of such a condition of things must be always uncertain till after the abdominal cavity has been opened. In the two last cases coming under my care the tumour was supposed to be an inflamed ovarian cyst, the true nature of the trouble being only ascertained at the time of operating. Since the diagnosis must always be doubtful, I should not hesitate to advise an operation. If the diagnosis be wrong, and the swelling prove to be an ovarian cyst, it can only be cured by removal; while if the diagnosis be right, and the swelling prove to be a large hæmatocele in Douglas's pouch, the safest way of dealing with it and preventing secondary complications will be by clearing out the mass. It is true that if left alone blood might be gradually absorbed, but I have seen a case in which its presence has caused the coils of intestine roofing it over to become matted together in a regular ball by dense adhesions which could not fail to be a source of subsequent danger from obstruction.

\section{Operation when the Tube is Ruptured into the} Peritoneal Cavity.

This is so simple that much need not be said about it. When the peritoneum is reached from the abdominal incision its dark colour at once shows that blood is beneath it. When opened a large quantity of blood and clot immediately pours out. The left hand passed quickly to the pelvis feels for the uterus, this forming a landmark; the ruptured sac can be at once grasped and drawn upwards, and a pair of long pressure clamps put upon either side of it, to be replaced by ligatures when the blood clot has been removed and a better view obtained.

All danger from further bleeding being over, attention is paid to the removal of the blood clot, which is often collected in enormous quantity in the abdominal cavity. This is best removed by a thorough flushing. A long glass irrigation tube is passed to the bottom of Douglas's pouch, and as the water flows back in a regurgitant stream the left hand is employed 
in gently moving the intestines so that all clot entangled between their folds is removed. When the water comes back clear from the pelvis the tube should be shifted to the kidney regions, and then below and a little behind the liver; also the pouch between the uterus and the bladder must be remembered. When the water comes back clear the flushing is complete. A long glass tube is passed down to the bottom of Douglas's pouch, and whilst the stitches are being introduced a small indiarubber tube passed down to the bottom of the glass tube is made to act as a siphon, quickly drawing off the accumulated water. The stitches are now all tightened, the glass tube removed, and the abdomen permanently closed, for $I$ am well satisfied that in most cases further drainage is not required.

\section{Operation when the Rupture is into the Broad}

Ligament or Limited to Douglas's Povch.

In these cases considerable caution must be exercised in making the abdominal incision, especially towards the lower angle, for the omentum and intestines may be adherent to the parietal peritoneum in this situation, and thus in danger of being wounded. It is best, therefore, to make a little opening through the parietal peritoneum in the upper angle sufficient to introduce the finger, which can be used as a guide for completing the peritoneal incision. On opening the abdominal cavity in all these cases I have found the condition nearly identical. The omentum is adherent, plastered like a lid over the pelvic contents, the free border being apparently adherent both to the anterior abdominal parietes and to the upper part of the uterus. The first step is to separate this. If at all firmly adherent it is better to trace it up till the free portion is found; it can here be ligatured in segments, the large clamp being placed below the ligatures is cut across. When this has been done the summit of the uterus can be made out. It is adherent behind to some coils of intestine. Upon gently separating these some black blood suddenly wells up, and a quantity of dark clot can be seen. This blood and clot is sometimes within a sort of cyst lining the interior of Douglas's pouch, or contained between the layers of the broad ligament. In one case (III) I was able to shell out this cyst-like body intact, but it could not be definitely ascertained what structure formed the cyst, whether it was the remains of an expanded tube, or simply the outer surface of the blood clot become firm and fibrinous. In most cases, forming part of the roof of the blood sac, is the thickened remains of the ruptured tube. The blood below is simply bounded by the structures forming Douglas's pouch, the clot being black and soft in the centre, but firmer towards the outer portions where it is adherent to the peritoneal lining of the pouch. The great bulk of the clot can be scooped out with the hand, and the remainder with more or less ease stripped off with the finger tips, or with the gentle use of the spoon. In three of my cases the fotus was found in this clot. After the clot has been scooped away as much as possible, the cavity can be well flushed. At this period of the operation, or earlier if it should appear more convenient, the remains of the tube, after being ligatured on either side, can be cut away. The question of employing a drainage tube is rather a nice one. In Cases II, III, v, vi it was used. Hitherto I have been guided by the condition of the cavity. If all the clot has been well washed away, I do not use it. On the other hand I have thought it safer to do so where there has been much ragged tissue left behind.

Extrauterine Fotation with a Living Fotus.

Cases in which development goes on till the fotus quickens are comparatively rare. Out of 14 cases of operation, I have only had one in which the child was alive. The child was between the sixth and seventh month, and the case was the only fatal one in the series.

\section{Treatment.}

There seem to be three courses open to the surgeon : 1. To operate at once, and remove the foetus.

2. To wait till near the natural time with a view to removing a living child.

3. To wait till the death of the child after its full time.

In deciding the best line of action, some surgeons are in- fluenced not solely by what is safest for the mother, but they also take into consideration the life of the child. Holding in respect the humanity which thus influences some operators, 1 must confess that my own decision would entirely turn on which course would involve the least risk to the mother's life. In all operations undertaken during the vitality of the fœtus the chief danger is from hæmorrhage which arises from the detachment of the placenta. This may be avoided in some cases by leaving the placenta intact, as will be subsequently discussed. Occasionally the pelvic brim is so plastered over as to make the removal of the fœtus impossible without detaching the placenta.

The rarenes 3 of these cases makes it impossible for any single operator to decide from his own experience as to the safest time for undertaking an operation, so that sufficient data upon which to found a decision can only be obtained from the accumulated experience of many operators. The most recent set of cases to which my attention has been called is that tabulated by Mr. Bland Sutton, whose accurate and careful work can invariably be relied on. Mr. Sutton records 29 cases of operation. In 16 of these laparotomy was performed after the death of the fotus at about full time. The remaining 13 cases were operated on between the seventh and ninth month, the child being alive. The results are remarkable and instructive. The 16 cases in which the operation was performed after the death of the fotus all recovered, while in the 13 operated upon whilst the child was alive, 8 died. Of the 13 children only 2 are mentioned as having lived; the remainder for the most part dying within a few hours.

If further experience confirms these valuable statistics there can be no question as to the relative danger of the two procedures, and that the surgeon, unless untoward complications are present, should defer operation till after the death of the fœetus. Nevertheless, he should be prepared to deal with the living child should necessity arise.

Operation when the Fotus is Alive.

After opening the abdomen and exposing the fotal sac the first question for decision is as to whether or not the placenta should be removed. Undoubtedly one of the chief causes of fatality is the severe hæmorrhage that such a removal entails. It must be remembered that this placental attachment may be widely spread, being plastered on to the intestines or other vital structures, from which it cannot be roughly torn without the risk of damage, or parts of it may be so deeply situated as to be difficult of access. The conditions after its removal are very different to when expelled from the interior of the uterus, for there the uterine contraction at once closes the open sinuses, arresting bleeding. With our present knowledge the best practice appears to be to remove the fœtus, and to divide the cord close to the placenta, leav ing this structure intact and untouched. Of course it can be understood that the fœetus might be so situated that it. would only be accessible by detaching, or partly detaching, the placenta. In these circumstances the surgeon may be driven, as the only means of arresting the bleeding, to remove the placenta as rapidly as possible, applying sponge or gauze pressure to the denuded surface. Assuming the surgeon has been so fortunate as to remove the fœtus without. exciting any violent placental bleeding, leaving the placenta. intact, a further question arises as to what should be done with regard to closing the wound. Dr. Champneys, in his historic case, thought it desirable to bring the cord, which had not been entirely removed, into the lower angle of the wound, and fixed it there. Other operators have cut the cord off short, and put in a drainage tube, while some completely close the abdominal wound without drainage. In the single case fallen to my experience (Case $x$ ) I adopted the latter procedure. The patient died, and from a careful consideration of the conditions found at the post-mortem examination, although, of course, it cannot be said that a drainage tube would have saved the patient, it certainly, as matters turned out, would have afforded her a better chance, and, should I meet with a second case under similar circumstances, I should employ drainage.

In my case, after removing the fœtus, it would not have been practicable to draw up the sac, and stitch it to the lower angle of the wound. If this should be possible, it would pro- 
bably be the best method of treatment, but, failing this, I should pass a drainage tube to the bottom of the sac, and
then close the sac as accurately as possible on either side of the tube.

CASE I.-L. J., a married woman, aged 43 , three children, last born two years ago,admitted to St. Bartholomew's hospital,Feb.,1892. Catamenia regubeginning of October some pain in the lower abdomen ; after that free loss of blood for seven days, accompanied with repeated attacks of pain which made her feel faint and sick. On December 4 th, whilst getting up, which made her feel faint and sick. On December 4th, whilst getting up, was suddenly seized with violent pain in the abdomen; this lasted for she had a second attack similar to the first, and was admitted to the hosshe had a second attack similar to the first, and was admitted to the hosmen considerably distended. Per vaginam nothing could be felt except a vague resistance high up. On opening the abdominal cavity a large amount of fluid blood and clot, amounting in all to some pints, poured out. In the right Fallopian tube was a gestation sac the size of a plover's egg. This had ruptured, and there was a bleeding blood clot protruding from its posterior surface through a hole the size of a threepenny piece. from its posterior surface through a hole the size of a threepenny piece. Three days later the patient passed a complete cast of the uterine cavity. Ten months after leaving the hospital she was safely delivered of a lealthy child. Recovery.

CASE II.-I. P., aged 27, admitted to the hospital November, 1892. She had two children, the last born five years ago. Catamenia regular. Two months ago she suffered severe pain in the abdomen, which recurred last month with almost constant pain, and an intermittent vaginal last month with almost constant pain, and an intermittent vaginal marked fluctuating prominence, the cervix was fixed, and all the pelvic marked fluctuating prominence, the cervix was fixed, and all the pelvic remained quiet in the hospital without any increase of the swelling for remained quiet in the hospital without any increase of the swelling for a abdomen, and the swelling was found to have considerably increased. On opening the abdomen, the omentum was adherent beneath the in cision. On detaching it, its under surface was adherent to the small intestine, and was plastered over the tumour. On separating this, the clot, apparently of recent origin. Below this and connected with it wa a second cavity about the same size which contained a small quantity of recent blood, but was chiefly filled with partially decolorised blood clot. The cavity was obviously intraperitoneal, being bounded above by the omentum and small intestines in front by the uterus, and below by that their exact position could not be made out. The cavity was washed out, and the adherent blood clot removed by a Volkmann's spoon. A darge quantity of dark bloodstained fluid was drawn off. On examining the removed blood clot, no fotus could be found, but some membranous substance which appeared to be placental. The patient recovered.

CASE IIr.-E. C., aged 3o, admitted into the hospital, June, r893 Married eight years, no children. Menstruation commenced at 20 , and a constant blood-stained discharge for nearly a montl. She was then seized during the night with a severe cramp-like pain, the whole abdomen becoming swollen and tender. She had two similar attacks at intervals of a fortnight. On each occasion she became very faint. On admis sion she looked healthy and the breasts showed no signs of activity : nothing could be felt by the abdomen: per vaginam the whole pelvic excavation was occupied by an elastic swelling. She remained at rest in bed for a fortnight; she then had another severe attack of pain and the glued over the pelvis. On separating this a red vascular-looking tumour appeared. Running over its surface was a greatly-dilated Fallopian tube The tumour sac was adherent to the rectum behind, and to the bladder and uterus to the right and in front. The adhesions were easily separated by the finger tips and there was considerable bleeding. The finger could pedicle was obtained, consisting apparently of the thickened tube and a portion of the broad ligament. The cyst was the size of a large fist, and a fotus 5 inches in length was found lying in Douglas's pouch, probably extruding from the cyst in the process of extraction. After removing the cyst the boundaries of the cavity were clearly seen. Behind was the rectum, in front the posterior surface of the broad ligament, and on the inner side the uterus. Nearly at the bottom of this cavity was a small circular opening going into the rectum, obviously produced by softening and ulceration. With great difficulty this was closed with a couple of stitches. A drainage tube was passed to the bottom of the cavity, and. after the flushing, the abdominal wound closed. For over a week gas but by the tenth day, the fluid having become clear, the drainage was
removed. The patient recovered.

CASE IV.-A. M., aged 34 admitted to the hospital at the end of January, 1894. She had had five children, the youngest aged only 5 months. After her last confinement she had several rigors. She has had two considerable floodings at intervals of a month. On admission she looked ill, but had a good pulse. Rising to a level with the navel was a smooth swelling ; per vaginam the whole pelvic excavation was occupied by an elastic tumour. hard in places. The uterus was lying in front and was nearly found lying in Douglas's pouch; it was readily the abdomen the sac was drawn up, but the hæmorrhace was so severe that an elastic ligature was slipped as far as possible below the sac. In doing this the cyst ruptured. much dark blood clot extruding; after this it was possible to transfix and tie a broad pedicle. The sac was cut off, the abdomen flushed and closed without drainage. On examination the cyst was thick-walled and covered by peritoneum, and the tube spread over it, the fimbriated extremity being last. When the tumour was cut into it contained blood CASE V.-M. Y., aged 4r, admitted November, 1894 ; ten children, the last ten months previously. Last regular period in August. For some pain. pain. Per vaginam an elastic tumour is felt behind the uterus. On opening the abdomen a coil of the sigmoid flexure formed the boundary of the tuinour. On separating this a sac was found in Douglas's pouch conmembranous substance found to be placental. All clot and membrane was removed, and a drainage tube used for fourteen days. The patient recovered.

CASE vI.-E. : B., aged 27, admitted November. 1894; three children, the last 13 months previously. Confinements all natural. Last period three months ago, very profuse. Three weeks later How reappeared, and some severe abdominal pain and vomiting, and two or three similar attacks since. A tumour was noticed soon after the first attack of pain. On admission she was very pale and restless; some albumen; breasts On admission she was very pale and restless; some albumen; breasts inactive. An ill-defined tender tumour was felt, rising high in the left pied by a tender, slightly elastic swelling. Operation.-On opening the pied by a tender, slightly elastic swelling. Operation.-On opening the
abdomen the omentum was plastered over the pelvis, and adlierent by
its free end to the bladder. It was tied and divided. The right tube then came into view. In its centre it was dilated into a hard swelling the then came into view. In its centre it was dilated into a hard sweling the size of a bantam's egg. Below and continuous with this was a tumour. Apparently this was between the layers of the broad ligament, and of the
size of a cocoanut. On opening this about a couple of pounds of dark size of a cocoanut. On opening this about a couple of pounds of dark
black coagula was removed, and the cavity sponged and washed out, a black coagula was removed, and the cavity sponged and washed out, glass diainage tube inserted, and the opened cavity closed around it with silk sutures, and the abdomen closed

CASE viI.-A. B., aged 36 . admitted January, r895. Married three years. never pregnant; last period six weeks ago. Ten days before admission she was kicked by her lusband, which was followed by an attack of severe abdominal pain. On the day of admission she had a sudden
paroxysm of pain whilst walking in the street; she fainted, and was paroxysm of pain whilst walking in the strcet; she fainted, and was carried to the hospital. On opening the abdonien a large quantity of blood poured out, together with a mass of clot, in which was a fotus about an inch long. The left tube was found dilated into a sac, which had ruptured in its upper and back part. It was tied and removed. Cavity flushed, but no drainage. On the fourth day the patient had pletely relieved by a temporary enterotomy. The patient recovered.

CASE VIII.-B. Y., aged 3r, admitted February, r895. Three children, the youngest 2 years oid. Menstruation regular. A fortnight ago she was truck on the abdomen. This was followed by acute abdominal pain. The pain recurred three days ago, since when she has had frequent vomiting. On admission she looked ill, and was very restless. The swollen and tender, both flanks dull. Per vaginam, the cervix was pressed against the symphysis. After ten days in bed all tenderness had gone. and she was about to leave the hospital, when she had the omentum was found tacked down to the right side of the pelvis. On cutting this across a large quantity of fresh blood welled up from the pelvis. The right tube large quantity of fresh blood welled up from the pelvis. The right tub recent rent was visible. Douglas's pouch was roughened, and covered recent rent was visie. Douglas's pouch was roughened, andosed without drainage. In the clots was found an ovum the size of a hen's egg. It out drainage. In the clots was found an ovum the size of a hen's egg. It an embryo half an incl long. showing the primary cephalic vesicles.

an embryo half an incl long. Showing the primary cephalic vesicles. CASE IX.-A. E., aged 29, admitted September, 1895 : married ten years and attacks of sickness and faintirg; lately has passed some offensive blood clots. Per vaginam, an irregular elastic swelling was felt behind the uterus. On opening the abdomen a simple broad ligament cyst was shelled out. Douglas's pouch was then found to be full of partly disorganised blood clot, which seemed to have extended from a cavity in the broad ligament. This was cleared out, leaving a jagged edge. Microage. The patient recovered.

CASE X.-M. M., the mother of seven children, the last twins, born eight years ago, admitted May, 1895. Regular till the previous November, since which time she has had irregular bleedings. A week previous to admission she had a severe attack of pelvic pain with fainting. On admission, there was marked tenderness over the whole abdomen, and a
tumour rising half way to the navel. The tumour occupied all the pelvic excavation. On opening the abdomen, the tumour was felt lying behind the uterus, and apparently between the layers of the broad
ligament. Its upper surface was covered by densely adherent omentum ligament. Its upper surface was covered by densely adherent omentum and intestine. On dissecting these off with much difficulty, the swelling was found to extend completely across the pelvis from one side to the other, and raising upwards the peritoneum of Douglas's pouch. Its appearance was that of a gravid uterus. being covered with hypertrophied muscular
fibres and dilated veins. The sac was opened. and a living foetus of about fibres and diluted veins. The sac was opened. and a living fotus of about
six montlis was removed. The placenta was firmly adherent to the walls of the sac. At one part a portion of this was accidentally detached, and there was a small amount of dark venous oozing, otherwise there was no
bleeding. The edges of the sac were united with silk sutures, but owing bleeding. The edges of the sac were united with silk sutures, but owing
to the depth of the lower portion not very satisfactorily; the abdomen to the depth of the lower portion not very satisfactorily; the abdomen
was closed without drainage. The patient died on the third day, the was closed without drainage. The patient died on the third day, the
following being an extract from Mr. Berry's notes on the post-mortem examination: "The uterus three times the normal size: belind and firmly attaclied to it was a transversely elongated oval sac in the broad ligament. Anteriorly it was smooth, and in contact with the round ligament. an inch thick. The sac was closed with silk sutures everywhere except
at the lowest corner. Douglas's pouch was nearly obliterated. The at the lowest corner. Douglas's pouch was nearly obliterated. The a portion of it being detached; the whole of it was soft and friable and beginning to decompose. The sac contained some blood-stained fluid some of which had leaked into the peritoneal cavity."

Eight of the cases recorded were admitted under the care o my colleague Dr. Champneys, the remaining two under 
Dr. Griffith being transferred to me for operation, and it is with their kind permission that I am able to publish the cases. I would also wish to thank Mr. Bruce Clarke for his valuable assistance in all the operations.

\section{THE PHYSIOLOGY OF THE CARBOHYDRATES.}

\section{a Rejoinder to Dr. Paton's Further Criticism.}

BY F. W. PAVY, M.D., LL.D., F.R.S., Consulting Physician to Guy's Hospital.

(Concluded from page 650.)

IT is impossible not to be struck by the direct manner in which the effects of the experimental injection of sugar into the subcutaneous tissue are revealed by the urine. In these experiments (transcribed in Epicriticism, p. 120), owing to the fact that absorption has to take place, the entry of sugar into the circulation can only be gradual. On the supposition that sugar slowly entering the circulation can be conveyed to the systemic capillaries for destruction without accompanying elimination by the urine this slow absorption would favour the escape of the urine from being influenced.

In a foot-note to his reply (p. 727) Dr. Paton writes "The presence of sugar in the blood in undiminished amount during starvation is a fact strongly opposed to Dr. Pavy's views." I do not concede that authority exists for the use of the words "in undiminished amount," and would ask whether there are any grounds for saying that the sugar of the blood does not, during starvation, participate in the shrinkage of material generally which occurs throughout the system? But with this reservation I admit the statement to be a statement of fact, but so far from being opposed to my views it lends support to them. Irrespective of food, there is always present in the blood a certain limited amount of sugar, just as in all the various tissues of the body. In the one case, as in the other, this certain amount of sugar may, I consider, be looked upon as standing in the position of a constitutional ingredient, and it is not necessary to look to the liver for its source. Operations involving building up and breaking down are everywhere being carried on in the body, and the power of self-maintenance in the presence of constant change is associated with these operations. If, however, a source for the sugar of the blood is to be looked for outside the blood itself, why may we not look upon it as derived from other structures, especially the muscles, instead of from the liver in particular? At the moment of death the position of the liver as regards sugar does not, as was formerly supposed owing to changes due to post-mortem effects, differ from that in other parts of the body. Indeed, the statement appears justifiable that there is ordinarily a higher percentage of sugar in muscle than in liver, while the bulk of the former in the body is considerably greater than that of the latter.

Corresponding with the amount of sugar naturally existing in the blood there is a certain amount of sugar encountered in the urine, but this amount is too small to have any significance when viewed in relation to the question of the physiological application of carbohydrate matter within the system.

Since sugar that is permitted to enter the general circulation shows itself in the urine, we are, by arguing backwards, forced to the conclusion that the amount of sugar in normal urine being so small sugar cannot reach the general circulation under normal conditions in quantity sufficient to be compatible with the theory that what is present has anything to do with a functional transit of the carbohydrate matter of our food to the tissues for destruction. Looking at all the facts before us, I do not consider it possible to conclude otherwise than that a close harmony exists between the state of the urine and that of the blood. Can it be thought that sugar is constantly reaching the blood for transit to the tissues without rendering itself apparent by its effect upon the urine? No insignificant amount as I have elsewhere shown, wopld have to be transmitted through the circulation if our carbohydrate matter were thus conveyed to the tissues. The teaehings of diabetes show in an unequivocal manner that ingress for transit cannot take place without sugar appearing in proportionate amount in the urine. To keep sugar out of the urine it must be kept out of the blood. In health the carbohydrate matter of the food produces no effect upon the blood of the general circulation, and consequently none upon the urine. The blood of the portal system contains an amount of sugar which fluctuates in accordance with the amount of carbohydrate ingested; the blood of the general circulation does not. In fact, so far as I am aware, no one contends that it does; if it did the amount of sugar in the urine under normal conditions would fluctuate as happens in diabetes.

It is then the power-call it assimilative or what you will - - possessed by the healthy individual of disposing of carbohydrate matter before it has an opportunity of entering the general circulation, which, by keeping the blood free from sugar derived from the food, prevents the urine from being influenced by the ingested carbohydrates. Now this power in the healthy subject is not without its limits, and a point may be reached at which the ingestion of carbohydrate in a soluble form such as sugar, especially if taken during fasting when absorption is rapid, is attended by the appearance of sugar in the urine. In diabetes there is either a loss or a greater or less diminution of this power, with the result that carbohydrate matter which can be ingested by a healthy person without producing any effect upon the urine leads to an escape of sugar in a degree proportionate to that in which the power is diminished.

In a particular case, for instance, it may happen that the assimilative power is only a little below what is normal, and here a considerable amount of carbohydrate food may be taken without being followed by saccharine urine; but if the amount exceed the limit at which the power stands, sugar is eliminated in proportion to the extent to which the point is exceeded, although such food would not in the healthy person give any evidence of effect upon the urine. And so it runs in different cases, until even what is considered the diabetic dietary, which, however, is not a dietary totally free from carbohydrate matter, but one containing a greatly diminished amount, is accompanied with a more or less notable elimination of sugar.

It is, in fact, made abundantly clear that we have to do in diabetes with the loss, in varying degree in different cases, of the power of assimilating or disposing of carbohydrate matter in such a way as to prevent its reaching a positionthe general circulation-in the form of sugar, whence, as common observation shows, it runs off as waste material in the urine. Everything depends, as regards the state of the urine, upon whether the assimilative power existing is taxed beyond its capacity or not. Up to a certain point, carbohydrate matter can be taken without any effect upon the urine being produced, whilst beyond the particular point sugar is discoverable.

No wonder these cases formerly proved perplexing to practitioner and patient. One day sugar was found and another day not. Variation in food affords the explanation, and a very little will suffice to determine the character of result encountered. It is indeed surprising the exactness observable from day to day, week to week, and even, it may be, for lengthened periods. As long as the patient is confining himself within a certain limit as regards carbohydrate matter taken, his urine presents a normal condition. Directly he exceeds the limit sugar is found, and found in proportion to the extent to which he has done so. We have a parallel condition to that of health, but with the difference that the power of assimilation--the power of preventing carbohydrate from reaching the general circulation as sugar, and thence the urine-exists in diminished amount.

All the facts before us in relation to food conspire to show that for the urine to be kept free from sugar, sugar must be kept out of the general circulation, and that it is the difference in the amount of power existing for the accomplishment of this that constitutes the difference between health and diabetes, and likewise the various degrees of the latter. When carbohydrate food is taken beyond the capacity of assimilating or properly disposing of it, that which is in excess of the capacity straightway passes or filters, as it were, through the system into the urine. Of necessity it must have been permitted to reach the general circulation for the purpose. Two or three hours quite suffice for determining the effect. With the greatest precision and con- 